\title{
Electrooptical Properties of Cylindrical Quantum Dots
}

\author{
P. SChillak* AND G. CZAJKOWSKI
}

University of Technology and Life Sciences, Kaliskiego 7, 85-789 Bydgoszcz Poland

\begin{abstract}
We show how to compute the optical functions (the complex electrosusceptibility tensor, dielectric tensor, electroreflection spectra) for semiconductor quantum dots exposed to a uniform static electric field in the growth direction, including the excitonic effects. The method uses the microscopic calculation of the quantum dot excitonic wave functions and energy levels, and the macroscopic real density matrix approach to compute the electromagnetic fields and susceptibilities. The electron-hole screened Coulomb potential is adapted and the valence band structure is taken into account in the cylindrical approximation, thus separating light- and heavy-hole motions. In the microscopic calculations, using the effective-mass approximation, we solve the 6-dimensional two-particle Schrödinger equation by transforming it into an infinite set of coupled second order 2-dimensional differential equations with the appropriate boundary conditions. These differential equations are solved numerically giving the eigenfunctions and the energy eigenvalues. Having them, we can compute the quantum dot electrooptical functions. Numerical calculations have been performed for an InGaAs quantum dot with a constant electric field applied in the growth direction. A good agreement with experiment is obtained.
\end{abstract}

PACS numbers: 73.21.La, 78.30.Fs, 78.67.Hc, 78.67.--n, 78.90.+t

\section{Introduction}

Optical properties of lower-dimensional structures are determined by the quantum size effect and excitons. The confinement of quasi-particles in a nanostructure leads to enhancement of the oscillator strength of excitons and thus to enhancement of excitonic effects. Calculations of the optical properties are quite complicated since a separation of the center-of-mass motion and the relative motion is not possible, and one has to solve a complicated boundary problem in a 6 -dimensional configuration space. Therefore various approximations have been proposed (for a review see, for example [1] and references therein).

In the present paper we consider a quantum dot of cylindrical shape, with the symmetry axis $z$ (the growth direction), and with infinite hard wall potentials for electrons and holes located in the $x y$ plane at the radius $R$. Such a quantum dot is also called a quantum disk (QDisk), see for example [2] and references therein. We compute the electrooptical functions of QDisks when an electric field $\boldsymbol{F}$ is applied in the $z$-direction, taking into account the electron-hole Coulomb interaction and the confinement effects. The presented method is a generalization of the method described in previous papers $[3,4]$.

To obtain the linear optical response we apply the real density matrix approximation (RDMA) $[5,6]$ and solve a set of coupled equations (constitutive equations CEs) for two-point correlation functions $\mathcal{Y}_{\mathrm{H}}$ and $\mathcal{Y}_{\mathrm{L}}$ for the

* corresponding author; e-mail: Piotr.Schillak@utp.edu.pl heavy-hole exciton $(\mathrm{H})$ and the light-hole exciton (L), and Maxwell's field equation. The functions $\mathcal{Y}_{\mathrm{H}}, \mathcal{Y}_{\mathrm{L}}$ give the total polarization

$$
\begin{aligned}
& \mathcal{P}(\boldsymbol{R}, t)=2 \int \mathrm{d}^{3} r\left[\boldsymbol{M}_{\mathrm{H}}^{*}(\boldsymbol{r}) \mathcal{Y}_{\mathrm{H}}(\boldsymbol{r}, \boldsymbol{R}, t)\right. \\
& \left.\quad+\boldsymbol{M}_{\mathrm{L}}^{*}(\boldsymbol{r}) \mathcal{Y}_{\mathrm{L}}(\boldsymbol{r}, \boldsymbol{R}, t)\right],
\end{aligned}
$$

where $\boldsymbol{M}$ is the dipole density appropriate to the considered interband transitions. The above equation with the constitutive equations connects the polarization with the electric field of the propagating wave. Using the long-wave approximation we then compute the effective QDisk susceptibility tensor, the dielectric tensor and, finally, the optical functions.

\section{Eigenfunctions, eigenenergies, and optical functions}

We compute the optical linear response to a linearly polarized incident wave. First we determine the eigenfunctions $\Psi$ of the QDisk Hamiltonian. We assume the eigenfunctions in the form

$$
\begin{aligned}
& \Psi\left(z_{\mathrm{e}}, z_{\mathrm{h}}, \boldsymbol{\rho}_{\mathrm{e}}, \boldsymbol{\rho}_{\mathrm{h}}\right) \\
& \quad=\sum_{n} \psi_{n_{1} m_{1}}^{\mathrm{e}}\left(\boldsymbol{\rho}_{\mathrm{e}}\right) \psi_{n_{2} m_{2}}^{\mathrm{h}}\left(\boldsymbol{\rho}_{\mathrm{h}}\right) f_{n}\left(z_{\mathrm{e}}, z_{\mathrm{h}}\right),
\end{aligned}
$$

$\psi^{\mathrm{e}, \mathrm{h}}(\boldsymbol{\rho})$ being the known eigenfunctions with the corresponding one-particle eigenenergies $\varepsilon^{(\mathrm{e}, \mathrm{h})}$ of the electron (hole) motion in a cylindrical quantum well, and $f_{n}\left(z_{\mathrm{e}}, z_{\mathrm{h}}\right), n=\left(n_{1}, m_{1}, n_{2}, m_{2}\right)$ unknown functions to be determined; $z_{\mathrm{e}}$ and $z_{\mathrm{h}}$ are the carriers (electron- and hole) coordinates in the $z$-direction, respectively. The functions $f_{n}$ and the total energy $\varepsilon$ obey an infinite system of 2-dimensional differential equations 


$$
\begin{aligned}
& {\left[\varepsilon_{n_{1} m_{1}}^{(\mathrm{e})}+\varepsilon_{n_{2} m_{2}}^{(\mathrm{h})}+\mathcal{E}\left(z_{\mathrm{e}}-z_{\mathrm{h}}\right)-\frac{\mu_{\|}}{m_{\mathrm{e} z}} \frac{\partial^{2}}{\partial z_{\mathrm{e}}^{2}}\right.} \\
& \left.-\frac{\mu_{\|}}{m_{\mathrm{h} z}} \frac{\partial^{2}}{\partial z_{\mathrm{h}}^{2}}\right] f_{n}+\sum_{n^{\prime}} V_{n}^{n^{\prime}} f_{n^{\prime}}\left(z_{\mathrm{e}}, z_{\mathrm{h}}\right)=\varepsilon f_{n},
\end{aligned}
$$

$\mathcal{E}$ is the rescaled strength of the applied electric field, $m_{\mathrm{e}, \mathrm{h} z}$ the respective effective masses, $\mu_{\|}$- the in-plane reduced mass, and $V_{n}^{n^{\prime}}\left(\left|z_{\mathrm{e}}-z_{\mathrm{h}}\right|\right)$ are the Coulomb interaction matrix elements between the in-plane confinement eigenfunctions.

\section{Results}

We performed numerical calculations for InGaAs (disk)/GaAs (barrier) QDisks, having in mind the experimental results by Oulton et al. [7]. We can restrict the number of functions $f_{n}$ used, obtaining a good convergence [4]. The results for the eigenvalues are illustrated
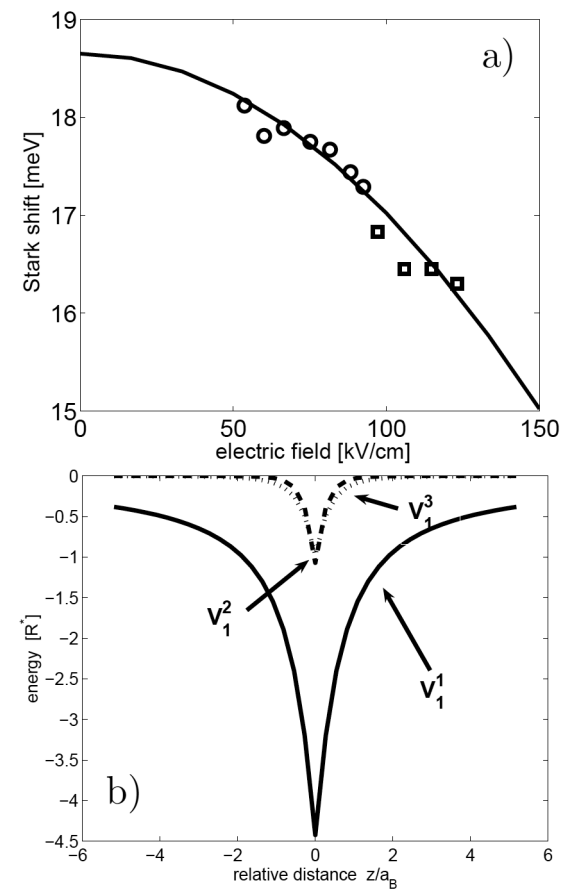

Fig. 1. (a) The energy shift of the excitonic ground state for an InGaAs/GaAs QDisk, theory (solid line) and experimental results by Oulton et al. (symbols) [7]. The disk height $L_{z}=6.2 \mathrm{~nm}$ and an effective disk radius $R=28 \mathrm{~nm}$ to fit the experiments. (b) The effective potentials for the heavy-hole exciton in an InGaAs/GaAs QDisk.

in Fig. 1a. We observe the Stark shift for the ground state energy and a good agreement with the experimental results. The functions $V_{n}^{n^{\prime}}$ play the role of the effective potentials. Some of them are displayed in Fig. 1b. We see that the main contribution results from the diagonal elements. The applied electric field changes the shape of the eigenfunctions as shown in Fig. 2 for the ground state.
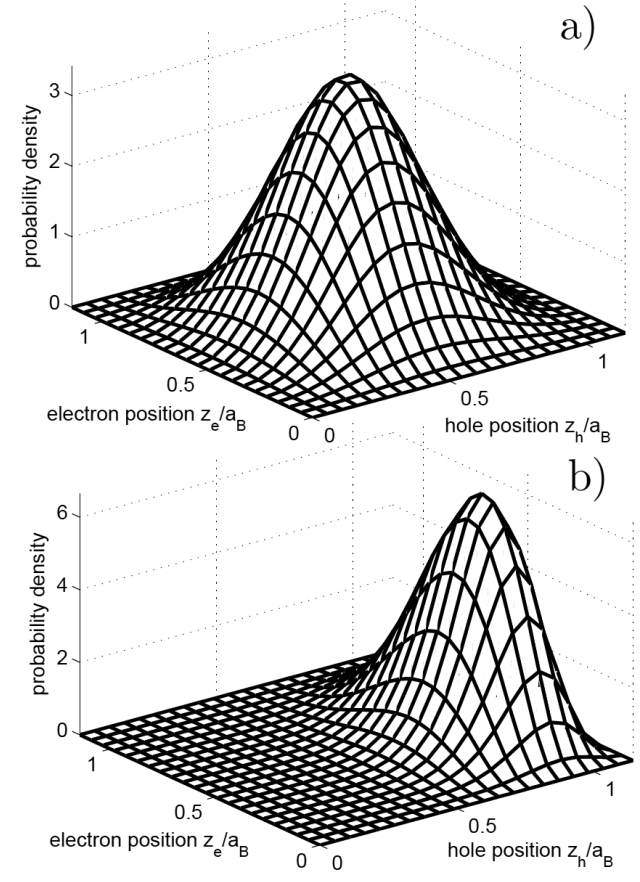

Fig. 2. The probability density projection on the $\left(z_{\mathrm{e}}, z_{\mathrm{h}}\right)$ subspace (a) without the electric field, (b) with the applied electric field $F=20 F_{\mathrm{I}}, F_{\mathrm{I}}$ is the ionization field.

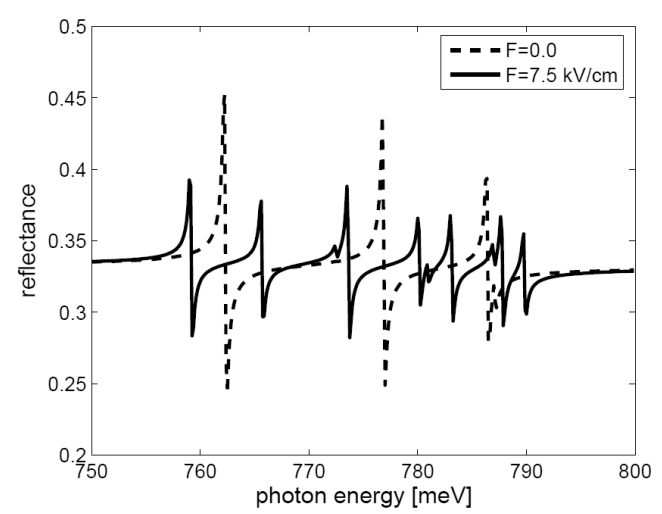

Fig. 3. Normal incidence reflectance spectra of a medium with distributed quantum disks for two values of the applied electric field.

The so obtained eigenfunctions (2) and the corresponding eigenvalues were then used to compute the functions $\mathcal{Y}_{\mathrm{H}}, \mathcal{Y}_{\mathrm{L}}$, and the corresponding polarization. Assuming a point-like shape for all relevant components of $\boldsymbol{M}$, we can compute the components of the QDisk average complex electrosusceptibility tensor and thus the dielectric tensor and, from them, the electrooptical functions. As example, the QDisk electroreflectance is shown in Fig. 3 for two values of the applied field. 


\section{Conclusions}

We have shown how electrooptical functions for quantum disks in the excitonic energy region can be computed with a high degree of accuracy. The main effect of the applied field is the red shift of the eigenenergies. The method can also be applied for other nanostructures of cylindrical symmetry (quantum rods, quantum wires, quantum rings, superlattices) and also for solving the so-called ABC problem (see for example $[5,6]$ ).

\section{Acknowledgments}

Numerical calculations were carried out at the Academic Computer Center in Gdańsk.

\section{References}

[1] S. Glutsch, Excitons in Low-Dimensional Semiconductors, Theory, Numerical Methods, Applications, Springer, Berlin 2004.
[2] G. Czajkowski, L. Silvestri, Central Europ. J. Phys. 4, 254 (2006)

[3] P. Schillak, G. Czajkowski, Acta Phys. Pol. A 112 , 301 (2007).

[4] P. Schillak, G. Czajkowski, Phys. Status Solidi C 6 , 415 (2009).

[5] A. Stahl, I. Balslev, Electrodynamics of the Semiconductor Band Edge, Springer, Berlin 1987.

[6] G. Czajkowski, F. Bassani, L. Silvestri, Rivista del Nuovo Cimento C 26, 1 (2003).

[7] R. Oulton, J.J. Finley, A.I. Tartakovskii, D.J. Mowbray, M.S. Skolnick, M. Hopkinson, A. Vasanelli, R. Ferreira, G. Bastard, Phys. Rev. B 68, 235301 (2003). 